\title{
Estimates of the $X(3872)$ cross section at a hadron collider
}

\author{
Eric Braaten, ${ }^{*}$ Li-Ping He®,$^{\dagger}$ and Kevin Ingles ${ }^{\ddagger}$ \\ Department of Physics, The Ohio State University, Columbus, Ohio 43210, USA
}

(Received 4 December 2018; revised manuscript received 7 October 2019; published 21 November 2019)

\begin{abstract}
The claim that the $X(3872)$ meson cannot be a charm-meson molecule because its prompt production cross section at hadron colliders is too large is based on an upper bound in terms of a cross section for producing charm-meson pairs. Assuming $X$ is sufficiently weakly bound, we derive an equality between the $X$ cross section and a charm-meson pair cross section that takes into account the threshold enhancement from the $X$ resonance. The cross section for producing $X$ is equal to that for producing $D^{* 0} \bar{D}^{0}$ integrated up to a relative momentum $k_{\max }=7.7 \gamma_{X}$, where $\gamma_{X}$ is the binding momentum of $X$. We also derive an order-ofmagnitude estimate of the $X$ cross section in terms of a naive charm-meson pair cross section that does not take into account the threshold enhancement, such as that produced by a Monte Carlo event generator. The cross section for producing $X$ can be approximated by the naive cross section for producing $D^{* 0} \bar{D}^{0}$ integrated up to a relative momentum $k_{\max }$ of order $\left(m_{\pi}^{2} \gamma_{X}\right)^{1 / 3}$. The estimates of the prompt $X$ cross section at hadron colliders are consistent with the cross sections observed at the Tevatron and the LHC.
\end{abstract}

DOI: 10.1103/PhysRevD.100.094024

\section{INTRODUCTION}

The discovery of a large number of exotic hadrons containing a heavy quark and its antiquark presents a major challenge to our understanding of QCD [1-10]. The first of these exotic hadrons to be discovered was the $X(3872)$ meson. It was discovered in 2003 in exclusive decays of $B^{ \pm}$ mesons into $K^{ \pm} X$ through the decay of $X$ into $J / \psi \pi^{+} \pi^{-}$ [11]. Its existence was quickly verified through inclusive production in $p \bar{p}$ collisions [12]. The $J^{\mathrm{PC}}$ quantum numbers of $X$ were eventually determined to be $1^{++}$[13]. Its mass is extremely close to the $D^{* 0} \bar{D}^{0}$ threshold, with the difference being only $0.01 \pm 0.18 \mathrm{MeV}$ [14]. This suggests that $X$ is a weakly bound $\mathrm{S}$-wave charm-meson molecule with the flavor structure

$$
|X(3872)\rangle=\frac{1}{\sqrt{2}}\left(\left|D^{* 0} \bar{D}^{0}\right\rangle+\left|D^{0} \bar{D}^{* 0}\right\rangle\right) .
$$

The $X$ can be produced by any reaction that can produce its constituents $D^{* 0} \bar{D}^{0}$ and $D^{0} \bar{D}^{* 0}$. In particular, it can be produced in high energy hadron collisions. The inclusive production of $X$ in $p \bar{p}$ collisions has been studied at the Tevatron by the CDF [12] and D0 [15] collaborations.

\footnotetext{
*braaten.1@osu.edu

the.1011@buckeyemail.osu.edu

*ingles.27@buckeyemail.osu.edu
}

Published by the American Physical Society under the terms of the Creative Commons Attribution 4.0 International license. Further distribution of this work must maintain attribution to the author(s) and the published article's title, journal citation, and DOI. Funded by SCOAP ${ }^{3}$.
The inclusive production of $X$ in $p p$ collisions has been studied at the Large Hadron Collider (LHC) by the LHCb [16], CMS [17], and ATLAS [18] collaborations. At a high energy hadron collider, $X$ is produced by the weak decays of bottom hadrons and by QCD mechanisms that create charm quarks and antiquarks. If $X$ is produced by the weak decays of bottom hadrons, its decay products emerge from a vertex displaced from the collision point. If $X$ is produced by QCD mechanisms, its decay products emerge from the collision point, so these mechanisms are referred to as prompt production. Cross sections for inclusive prompt production of $X$ have been measured by the CDF [12], CMS [17], and ATLAS [18] collaborations.

The substantial prompt production rate of $X$ at hadron colliders has often been used as an argument against its identification as a charm-meson molecule. This argument is based on an upper bound on the cross section for producing $X$ in terms of the cross section for producing the charmmeson pair $D^{* 0} \bar{D}^{0}$ integrated up to a maximum relative momentum $k_{\max }$ [19]. The estimate for $k_{\max }$ in Ref. [19] was approximately the binding momentum $\gamma_{X}$ of the $X$. In Ref. [20], it was pointed out that the derivation of the upper bound in Ref. [19] requires $k_{\max }$ to be of the order of the pion mass $m_{\pi}$ instead of $\gamma_{X}$. In this paper, we use the methods of Ref. [20] to derive equalities between the $X$ cross section and $D^{* 0} \bar{D}^{0}$ cross sections integrated up to $k_{\max }$. If we take into account the threshold enhancement due to the $X$ resonance, the value of $k_{\max }$ is $7.7 \gamma_{X}$. If we use a naive $D^{* 0} \bar{D}^{0}$ cross section without the threshold enhancement, the value of $k_{\max }$ is order $\left(m_{\pi}^{2} \gamma_{X}\right)^{1 / 3}$. The resulting estimates of the prompt cross sections are compatible with the measurements at the Tevatron and the LHC. 
The outline of this paper is as follows. In Sec. II, we describe some universal aspects of weakly bound S-wave molecules and the scattering of their constituents. In Sec. III, we present experimental upper and lower bounds on the cross sections for the production of $X$ at the Tevatron and the LHC. In Sec. IV, we discuss the theoretical upper bound on the prompt cross section for producing $X$ at hadron colliders derived in Ref. [19]. In Sec. V, we derive equalities between the $X$ cross section and a charm-meson pair cross section with and without the threshold enhancement from the $X$ resonance. In Sec. VI, we summarize our results and discuss their implications.

\section{BOUND S-WAVE MOLECULE}

If short-range interactions produce an S-wave bound state extremely close to a scattering threshold, the few-body physics has universal aspects that are determined by the binding momentum $\gamma_{X}$ of the bound state [21]. The binding energy is $\gamma_{X}^{2} / 2 \mu$, where $\mu$ is the reduced mass of the constituents. The momentum-space wave function in the region of the relative momentum $k$ below the inverse range has the universal form

$$
\psi_{X}(k)=\frac{\sqrt{8 \pi \gamma_{X}}}{k^{2}+\gamma_{X}^{2}} .
$$

The low-energy scattering of the constituents also has universal aspects determined by $\gamma_{X}$ through a simple function of the complex energy $E$ relative to the scattering threshold:

$$
f_{X}(E)=\frac{1}{-\gamma_{X}+\sqrt{-2 \mu E}} .
$$

This function has a branch cut along the positive $E$ axis and a pole at $E=-\gamma_{X}^{2} / 2 \mu$. The universal elastic scattering amplitude in the region of relative momentum $k$ below the inverse range is obtained by evaluating this function at energy $E=k^{2} / 2 \mu+i \epsilon$.

The analytic function $f_{X}(E)$ also gives the energy distribution from creation of the constituents at short distances. By the optical theorem, the distribution in the energy $E$ below the energy scale set by the range is proportional to the imaginary part of $f_{X}(E)$ :

$\operatorname{Im}\left[f_{X}(E+i \epsilon)\right]=\frac{\pi \gamma_{X}}{\mu} \delta\left(E+\gamma_{X}^{2} / 2 \mu\right)+\frac{\sqrt{2 \mu E}}{\gamma_{X}^{2}+2 \mu E} \theta(E)$.

There is a delta-function term at a negative energy from the production of the weakly bound molecule and a thetafunction term with positive energy from the production of the constituents of the molecule.

The universal energy distribution in Eq. (4) from the creation of the constituents at short distances is illustrated in Fig. 1. The delta-function term below the scattering

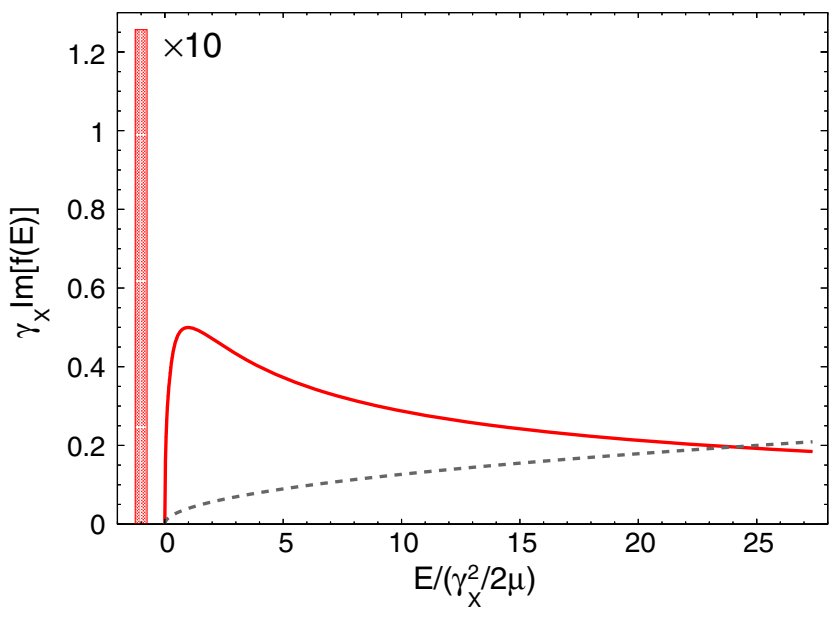

FIG. 1. Universal energy distribution $\operatorname{Im}\left[f_{X}(E+i \epsilon)\right]$ in Eq. (4) as a function of the energy $E$. The tall rectangle below the scattering threshold at $E=0$ represents the delta function from production of the bound state and should be multiplied by 10 . The dashed curve is the naive energy distribution in the absence of the resonance given by Eq. (5) with $\Lambda=5 \gamma_{X}$.

threshold at $E=0$ is represented by a tall rectangle centered at $E=-\gamma_{X}^{2} / 2 \mu$ which would have the correct area if it was multiplied by 10 . That area is equal to the area of the energy distribution above the threshold integrated up to the energy $20.2\left(\gamma_{X}^{2} / 2 \mu\right)$, which corresponds to a relative momentum $k_{\max }=4.49 \gamma_{X}$. The energy distribution above the threshold has a maximum at the binding energy $\gamma_{X}^{2} / 2 \mu$. For $E$ much larger than $\gamma_{X}^{2} / 2 \mu$, the universal energy distribution in Eq. (4) decreases as $E^{-1 / 2}$. This scaling behavior should continue up to the energy scale set by the range.

The naive energy distribution in the absence of the resonance has a form at low energy that can be obtained from Eq. (4) by omitting the delta-function term and by replacing the denominator $\gamma_{X}^{2}+k^{2}$ in the theta-function term by $\Lambda^{2}$, where $\Lambda$ is some momentum of the order of the inverse range:

$$
\operatorname{Im}\left[f_{\text {naive }}(E+i \epsilon)\right]=\frac{1}{\Lambda^{2}} \sqrt{2 \mu E} \theta(E) .
$$

The naive energy distribution is shown as a dashed line in Fig. 1. The actual energy distribution should cross over from the solid line to the dashed line, and it should therefore have a local minimum near $\Lambda^{2} / 2 \mu$. The area under the delta function from the bound state is equal to the area of the naive energy distribution integrated up to the energy $\left(3 \pi \gamma_{X} \Lambda^{2}\right)^{2 / 3} / 2 \mu$, which corresponds to a relative momentum $k_{\max }=\left(3 \pi \gamma_{X} \Lambda^{2}\right)^{1 / 3}$.

If the $X(3872)$ is a weakly bound charm-meson molecule, its constituents are the superposition of charm mesons in Eq. (1). We denote the masses of the charm mesons $D^{0}$ and $D^{* 0}$ by $M_{0}$ and $M_{* 0}$, respectively. The reduced mass of $D^{* 0} \bar{D}^{0}$ is $\mu=M_{* 0} M_{0} /\left(M_{* 0}+M_{0}\right)$. 
The range of the interactions between the charm mesons is $1 / m_{\pi}$, where $m_{\pi}$ is the pion mass. The corresponding energy scale $m_{\pi}^{2} / 2 \mu$ is about $10 \mathrm{MeV}$. This is comparable to the energy of the $D^{*+} D^{-}$scattering threshold, which is 8.2 MeV above the $D^{* 0} \bar{D}^{0}$ scattering threshold. The present value of the difference $E_{X}$ between the mass of the $X$ and the energy of the $D^{* 0} \bar{D}^{0}$ scattering threshold is [14]

$E_{X} \equiv M_{X}-\left(M_{* 0}+M_{0}\right)=(+0.01 \pm 0.18) \mathrm{MeV}$.

The central value in Eq. (6) corresponds to a charm-meson pair above the scattering threshold. The value lower by $1 \sigma$ corresponds to a bound state with binding energy $\left|E_{X}\right|=$ $0.17 \mathrm{MeV}$ and binding momentum $\gamma_{X}=18 \mathrm{MeV}$. The upper bound on the binding energy with $90 \%$ confidence level is $\left|E_{X}\right|<0.22 \mathrm{MeV}$.

Some qualitative aspects of the energy distribution illustrated in Fig. 1 have been observed by the Belle collaboration in the decays of $B$ mesons into $K D^{0} \bar{D}^{0} \pi^{0}$ [22]. The $D^{0} \bar{D}^{0} \pi^{0}$ invariant-mass distribution has a peak near the $D^{* 0} \bar{D}^{0}$ threshold. The energy resolution was not sharp enough to resolve the contributions from the narrow peak below the $D^{* 0} \bar{D}^{0}$ threshold from the $X$ bound state and the peak above the threshold from the $D^{* 0} \bar{D}^{0}$ and $D^{* 0} \bar{D}^{* 0}$ threshold enhancements. The difference between the fitted curve to the $D^{0} \bar{D}^{0} \pi^{0}$ invariant-mass distribution and the combinatorial background in Ref. [22] has a minimum at a $D^{0} \bar{D}^{0} \pi^{0}$ energy about $11 \mathrm{MeV}$ above the $D^{* 0} \bar{D}^{0}$ threshold. If we set $\Lambda^{2} / 2 \mu=11 \mathrm{MeV}$, we get an estimate for $\Lambda$ of about $150 \mathrm{MeV}$. This is consistent with $\Lambda$ being of order $m_{\pi}$.

\section{CROSS SECTIONS FOR $X$ AT HADRON COLLIDERS}

In this section, we summarize experimental results on the inclusive prompt cross sections for $X(3872)$ at the Tevatron and the LHC. We use them to obtain upper and lower bounds on the cross sections.

Within months of the discovery of the $X$ in $B$ meson decays by the Belle collaboration in September 2003 [11], its existence was confirmed by the CDF collaboration through inclusive production of $X$ in $p \bar{p}$ collisions at the Tevatron [12]. The $X$ was observed in the discovery decay mode $J / \psi \pi^{+} \pi^{-}$. Some of the $X$ events were produced from decays of bottom hadrons, with the ultimate decay products $\mu^{+} \mu^{-} \pi^{+} \pi^{-}$emerging from a displaced vertex. The majority of $X$ events were produced promptly, with the ultimate decay products emerging from the primary collision vertex. The prompt $X$ events presumably come from QCD production mechanisms. The CDF collaboration has reported ratios of the prompt cross sections for $X$ and $\psi(2 S)$ modulo the ratio $\epsilon_{\psi} / \epsilon_{X}$ of the efficiencies for observations of $\psi(2 S)$ and $X$ in their $J / \psi \pi^{+} \pi^{-}$decay modes [23]. The ratio $\epsilon_{\psi} / \epsilon_{X}$ is presumably closer to 1 than to 2 or $1 / 2$. In Ref. [19]
$\left(\mathrm{BGP}^{2} \mathrm{~S}\right)$, measurements of the prompt cross sections for $X$ and $\psi(2 S)$ by the CDF collaboration were used to obtain an estimate of the product $\sigma \mathrm{Br}$ of the prompt cross section for $X$ and its branching fraction into $J / \psi \pi^{+} \pi^{-}$in the region with rapidity $|y|<0.6$ and transverse momentum $p_{T}>5 \mathrm{GeV}$ :

Tevatron: $\sigma[X(3872)] \operatorname{Br}\left[X \rightarrow J / \psi \pi^{+} \pi^{-}\right] \approx(3.1 \pm 0.7) \mathrm{nb}$.

A loose lower bound on the cross section for $X$ can be obtained by using $\mathrm{Br}<1$.

The inclusive production of the $X$ in $p p$ collisions at the LHC has been studied by the LHCb [16], CMS [17], and ATLAS [18] collaborations. The CMS collaboration measured the product $\sigma \mathrm{Br}$ of the prompt cross section for $X$ and its branching fraction into $J / \psi \pi^{+} \pi^{-}$for $X$ with $|y|<1.2$ and $10 \mathrm{GeV}<p_{T}<30 \mathrm{GeV}$ at center-of-mass energy $7 \mathrm{TeV}[17]:$

$$
\begin{gathered}
\text { LHC: } \sigma[X(3872)] \operatorname{Br}\left[X \rightarrow J / \psi \pi^{+} \pi^{-}\right] \\
=(1.06 \pm 0.11 \pm 0.15) \mathrm{nb} .
\end{gathered}
$$

The prompt fraction was measured to be about $74 \%$ in the range $10 \mathrm{GeV}<p_{T}<50 \mathrm{GeV}$ [17].

In Ref. [24], we derived upper and lower bounds on the branching fraction $\mathrm{Br}$ for the $X$ bound state to decay into $J / \psi \pi^{+} \pi^{-}:$

$$
4 \%<\operatorname{Br}\left[X \rightarrow J / \psi \pi^{+} \pi^{-}\right]<33 \% .
$$

The loose lower bound $\mathrm{Br}>4 \%$ is derived from a recent measurement by the $B A B A R$ collaboration of the inclusive branching fraction for $B^{+}$into $K^{+}$plus the $X$ resonance feature [25]. The upper bound $\mathrm{Br}<33 \%$ is derived from measurements of branching ratios of $J / \psi \pi^{+} \pi^{-}$over other short-distance decay modes of the $X$.

Given the results for $\sigma \mathrm{Br}$ in Eqs. (7) and (8), a constraint on the branching fraction into $J / \psi \pi^{+} \pi^{-}$gives constraints on the prompt cross sections. A lower bound on the prompt cross section $\sigma$ can be estimated by decreasing the central value of $\sigma \mathrm{Br}$ by 1 standard deviation and then dividing it by the upper bound on $\mathrm{Br}$ in Eq. (9). A loose upper bound on $\sigma$ can be estimated by increasing the central value of $\sigma \mathrm{Br}$ by 1 standard deviation and then dividing it by the lower bound on $\mathrm{Br}$ in Eq. (9). Using the estimate in Eq. (7), the bounds on the prompt cross section at the Tevatron with $|y|<0.6$ and $p_{T}>5 \mathrm{GeV}$ are

$$
\text { Tevatron: } 7.3 \mathrm{nb}<\sigma[X(3872)]<95 \mathrm{nb} \text {. }
$$

Using the measurement in Eq. (8), the bounds on the prompt cross section at the LHC with $|y|<1.2$ and $10 \mathrm{GeV}<p_{T}<30 \mathrm{GeV}$ are 


$$
\text { LHC: } 2.6 \mathrm{nb}<\sigma[X(3872)]<31 \mathrm{nb} \text {. }
$$

In both Eqs. (10) and (11), the loose upper bound is more than 10 times larger than the lower bound.

In Ref. [26], ranges of prompt cross sections $\sigma$ for $X$ at the Tevatron and at the LHC were obtained by considering branching fractions in the range $2.7 \%<\mathrm{Br}<8.3 \%$ [27]. The lower ends of their ranges were about 5 times larger than our lower bounds on the cross sections in Eqs. (10) and (11). The differences come primarily from two sources. First, the lower end of their range for $\sigma$ was obtained from the central value of $\sigma \mathrm{Br}$ rather than from the value lower by 1 standard deviation. Second, the upper end of their range for $\mathrm{Br}$ was about 4 times smaller than our upper bound in Eq. (9). The smaller upper bound on $\mathrm{Br}$ was obtained by assuming that measurements of the branching fraction of $X$ into $D^{0} \bar{D}^{* 0}$ are dominated by the decay of the $X$ resonance into $D^{0} \bar{D}^{0} \pi^{0}$ and $D^{0} \bar{D}^{0} \gamma$ below the $D^{0} \bar{D}^{* 0}$ threshold and have a negligible contribution from the threshold enhancement in the production of $D^{0} \bar{D}^{* 0}$ above the threshold. This assumption is contradicted by measurements of the width of the $X$ from the $D^{0} \bar{D}^{* 0}$ decay mode, which are significantly larger than the upper bound on the width obtained by the Belle collaboration from the $J / \psi \pi^{+} \pi^{-}$decay mode [28].

\section{UPPER BOUND ON CROSS SECTION FOR $X$}

In this section, we present the upper bound on the inclusive prompt cross section for producing $X(3872)$ in Ref. [19]. We explain why the derivation of the upper bound requires the charm-meson pair cross section to be integrated up to a relative momentum of order $m_{\pi}$ instead of order $\gamma_{X}$, as apparently assumed in Ref. [19].

If $X$ is a charm-meson molecule with the flavor structure in Eq. (1), the inclusive cross section for producing $X$ can be expressed in terms of the same amplitudes as those in the inclusive cross sections for producing $D^{* 0} \bar{D}^{0}$ and $D^{0} \bar{D}^{* 0}$ [19]. The inclusive cross sections for producing $D^{* 0} \bar{D}^{0}$ and $D^{0} \bar{D}^{* 0}$ with small relative momentum $\boldsymbol{k}$ in the charmmeson-pair rest frame and the inclusive cross section for producing $X$ can be expressed as

$$
d \sigma\left[D^{* 0} \bar{D}^{0}\right]=\frac{1}{\text { flux }} \sum_{y} \int d \Phi_{\left(D^{*} \bar{D}\right)+y}\left|\mathcal{A}_{D^{* 0} \bar{D}^{0}+y}(\boldsymbol{k})\right|^{2} \frac{d^{3} k}{(2 \pi)^{3} 2 \mu},
$$

$$
d \sigma\left[D^{0} \bar{D}^{* 0}\right]=\frac{1}{\text { flux }} \sum_{y} \int d \Phi_{\left(D^{*} \bar{D}\right)+y}\left|\mathcal{A}_{D^{0} \bar{D}^{* 0}+y}(\boldsymbol{k})\right|^{2} \frac{d^{3} k}{(2 \pi)^{3} 2 \mu},
$$

$$
\begin{aligned}
d \sigma[X(3872)]= & \frac{1}{\text { flux }} \sum_{y} \int d \Phi_{\left(D^{*} \bar{D}\right)+y} \mid \int \frac{d^{3} k}{(2 \pi)^{3}} \psi_{X}(k) \\
& \times\left.\frac{\mathcal{A}_{D^{* 0} \bar{D}^{0}+y}(\boldsymbol{k})+\mathcal{A}_{D^{0} \bar{D}^{* 0}+y}(\boldsymbol{k})}{\sqrt{2}}\right|^{2} \frac{1}{2 \mu},
\end{aligned}
$$

where $\mu$ is the reduced mass of $D^{* 0} \bar{D}^{0}$. The sums over $y$ are over all the additional particles that can be produced. The amplitudes that appear in the cross section for $X$ in Eq. (12c) are the charge-conjugation-even superpositions of the amplitudes for producing $D^{* 0} \bar{D}^{0}+y$ and $D^{0} \bar{D}^{* 0}+y$. The momentum-space wave function for the $X$ in Eq. (12c) is normalized so $\int\left(d^{3} k /(2 \pi)^{3}\right)\left|\psi_{X}(k)\right|^{2}=1$. The differential phase space $d \Phi_{\left(D^{*} \bar{D}\right)+y}$ is that for a composite particle denoted by $\left(D^{*} \bar{D}\right)$ with mass $M_{* 0}+M_{0}$ plus the additional particles $y$. The mass of $X$ is sightly smaller than $M_{* 0}+M_{0}$ and the invariant mass of a charm-meson pair is larger than $M_{* 0}+M_{0}$, but the differences in the phase space integrals are negligible. Factors of 3 from the sums over the spin states of $D^{* 0}$ or $\bar{D}^{* 0}$ or $X$ are absorbed into the amplitudes $\mathcal{A}$. The phase-space integrals in Eqs. (12) are over the 3-momenta of the additional particles $y$, but the cross sections remain differential in the 3-momentum $\boldsymbol{P}$ of $\left(D^{*} \bar{D}\right)$. Thus the $D^{* 0} \bar{D}^{0}$ and $D^{0} \bar{D}^{* 0}$ cross sections in Eqs. (12a) and (12b) are differential in both $\boldsymbol{P}$ and $\boldsymbol{k}$, while the $X$ cross section in Eq. (12c) is differential only in $\boldsymbol{P}$.

In the expression for the $X$ cross section in Eq. (12c), there are interference terms between the amplitudes for producing $D^{* 0} \bar{D}^{0}+y$ and $D^{0} \bar{D}^{* 0}+y$. The interference terms are positive for some sets of additional final-state particles $y$ and negative for others. In high-energy hadron collisions, there are dozens or even hundreds of additional particles. The sum over the many additional particles $y$ gives cancellations that suppress the interference terms. The $X$ cross section in Eq. (12c) then reduces to the sum of a $D^{* 0} \bar{D}^{0}$ term and a $D^{0} \bar{D}^{* 0}$ term. At large transverse momentum, the hadronization of a $c \bar{c}$ pair is equally likely to produce $D^{* 0} \bar{D}^{0}$ and $D^{0} \bar{D}^{* 0}$, because the probability of a light quark or antiquark from a colliding hadron to become a constituent of one of the charm mesons is very small. The $D^{* 0} \bar{D}^{0}$ term and the $D^{0} \bar{D}^{* 0}$ term should therefore be equal, and the expression for the $X$ cross section can be reduced to

$$
\begin{aligned}
& d \sigma[X(3872)] \\
& =\frac{1}{\text { flux }} \sum_{y} \int d \Phi_{\left(D^{*} \bar{D}\right)+y}\left|\int \frac{d^{3} k}{(2 \pi)^{3}} \psi_{X}(k) \mathcal{A}_{D^{* 0} \bar{D}^{0}+y}(\boldsymbol{k})\right|^{2} \frac{1}{2 \mu} .
\end{aligned}
$$

The authors of Ref. [19] $\left(\mathrm{BGP}^{2} \mathrm{~S}\right)$ derived a theoretical upper bound on the cross section for producing $X$ in terms of a cross section for producing the charm-meson pair $D^{* 0} \bar{D}^{0}$. To derive their upper bound, $\mathrm{BGP}^{2} \mathrm{~S}$ first restricted the integral over the relative momentum in Eq. (13) to a region $|\boldsymbol{k}|<k_{\max }$ in which $\psi_{X}(k)$ differs 
significantly from 0 . They then applied the Schwarz inequality to that integral:

$$
\begin{aligned}
& d \sigma[X(3872)] \\
& \leq \int^{\prime} \frac{d^{3} k}{(2 \pi)^{3}}\left|\psi_{X}(k)\right|^{2} \cdot \frac{1}{\text { flux }} \sum_{y} \int d \Phi_{\left(D^{*} \bar{D}\right)+y} \\
& \quad \times \int^{\prime} \frac{d^{3} k}{(2 \pi)^{3}}\left|\mathcal{A}_{D^{* 0} \bar{D}^{0}+y}(\boldsymbol{k})\right|^{2} \frac{1}{2 \mu},
\end{aligned}
$$

where the primes on the integrals indicate restrictions to $|\boldsymbol{k}|<k_{\max }$. The first factor on the right side of Eq. (14) is the probability for the constituents of the $X$ to have relative momentum less than $k_{\max }$. Since this probability is less than 1 , they obtained the inequality

$$
\sigma[X(3872)]<\sigma\left[D^{* 0} \bar{D}^{0}\left(k<k_{\max }\right)\right] .
$$

The validity of this inequality hinges on the validity of restricting the integral in Eq. (13) to the region $|\boldsymbol{k}|<k_{\max }$.

In Ref. [19], $\mathrm{BGP}^{2} \mathrm{~S}$ did not give an unambiguous prescription for the maximum momentum $k_{\max }$ in the inequality in Eq. (15). They quoted the difference $E_{X}$ between the mass of the $X$ and the energy of the $D^{* 0} \bar{D}^{0}$ scattering threshold at that time as $E_{X}=-0.25 \pm 0.40 \mathrm{MeV}$. The central value corresponds to binding momentum $\gamma_{X}=22 \mathrm{MeV}$, and the value lower by $1 \sigma$ corresponds to $\gamma_{X}=35 \mathrm{MeV}$. In Ref. [19], BGP ${ }^{2} S$ chose $k_{\max }$ in the inequality in Eq. (15) to be $35 \mathrm{MeV}$. In a subsequent paper Ref. [29], whose authors included most of those of Ref. [19], an updated estimate $k_{\max }=20 \mathrm{MeV}$ was given. This is close to the binding momentum $\gamma_{X}=18 \mathrm{MeV}$ from the value of $E_{X}$ that is $1 \sigma$ below the central value in Eq. (6). The choice for $k_{\max }$ in both papers is consistent with the assumption that $k_{\max }$ is approximately $\gamma_{X}$, although this assumption was not stated explicitly in Ref. [19].

The conclusions of Ref. [19] were challenged in Ref. [20], which argued that the appropriate choice of $k_{\max }$ in the upper bound in Eq. (15) is of order $m_{\pi}$ instead of order $\gamma_{X}$. If short-range interactions produce an $\mathrm{S}$-wave bound state close to a scattering threshold, the momentumspace wave function in the momentum region below the inverse range has the universal form in Eq. (2). The normalization integral of the probability density $\left|\psi_{X}(k)\right|^{2}$ is dominated by $k$ of order $\gamma_{X}$. However the integral over $\boldsymbol{k}$ in Eq. (12c), whose integrand has only one factor of $\psi_{X}(k)$, is not dominated by $k$ of order $\gamma_{X}$. It has significant contributions from the region extending up to $k$ of order $m_{\pi}$, which is where the wave function $\psi_{X}(k)$ begins to fall faster than $1 / k^{2}$. Thus the derivation of the upper bound in Ref. [19] requires $k_{\max }$ to be of order $m_{\pi}$ instead of order $\gamma_{X}$, as was apparently assumed in Ref. [19].

In Ref. [19], $\mathrm{BGP}^{2} \mathrm{~S}$ estimated the cross section for charm-meson pairs with relative momentum $k$ at the Tevatron using the event generators HERWIG and PYTHIA to produce hadronic final states from $2 \rightarrow 2$ parton processes, primarily $g g \rightarrow g g$. This extremely inefficient method gave distributions at small $k$ with the behavior $k d k$. Their estimate for the theoretical upper bound on the prompt cross section for $X$ at the Tevatron obtained by inserting $k_{\max }=35 \mathrm{MeV}$ into Eq. (15) was $0.07 \mathrm{nb}$ using HERWIG and $0.11 \mathrm{nb}$ using PYTHIA. These cross sections are about 30 times smaller than the loose lower bound of $3.1 \mathrm{nb}$ given by the right side of Eq. (7). $\mathrm{BGP}^{2} \mathrm{~S}$ concluded that if the $X$ was a weakly bound charm-meson molecule, its formation from charm mesons at the rate observed at the Tevatron would be unlikely. Given that their $D^{* 0} \bar{D}^{0}$ cross section scaled as $k_{\max }^{2}$, the value of $k_{\max }$ would have to be larger than about $280 \mathrm{MeV}$ for the $D^{* 0} \bar{D}^{0}$ cross section calculated using PYTHIA to be above the lower bound on the $X$ cross section at the Tevatron in Eq. (10).

In Ref. [20], the cross section for charm-meson pairs with small relative momentum $k$ at the Tevatron was estimated using the event generator PYTHIA to produce hadronic final states from the $2 \rightarrow 3$ parton process $g g \rightarrow c \bar{c} g$. The distribution had the behavior $k^{2} d k$ in the region $k<m_{\pi}$. This behavior should be more accurate than the behavior $k d k$ obtained in Ref. [19], because the event generator, which has not been tuned to reproduce distributions in $k$, plays a smaller role in generating the distributions. Their estimate for the charm-meson-pair cross section at the Tevatron integrated up to a relative momentum $k_{\max }$ was

Tevatron: $\sigma_{\text {naive }}\left[D^{* 0} \bar{D}^{0}\left(k<k_{\max }\right)\right] \approx 0.03 \mathrm{nb}\left(\frac{k_{\max }}{35 \mathrm{MeV}}\right)^{3}$.

The subscript "naive" emphasizes that the cross section calculated using an event generator does not take into account the effects of the $X$ resonance. The theoretical upper bound in Eq. (15) is greater than the loose lower bound of $3.1 \mathrm{nb}$ given by the right side of Eq. (7) if $k_{\max }$ is greater than about $160 \mathrm{MeV}$. The authors of Ref. [20] concluded that the upper bound in Eq. (15) with $k_{\max }$ of order $m_{\pi}$ was compatible with the observed prompt cross section for $X$ at the Tevatron. The upper and lower bounds on the $X$ cross section at the Tevatron in Eq. (10) take into account the bounds on the branching fraction for $X \rightarrow J / \psi \pi^{+} \pi^{-}$in Eq. (9). The value of $k_{\max }$ would have to be larger than $220 \mathrm{MeV}$ for the naive $D^{* 0} \bar{D}^{0}$ cross section to be above the lower bound on the $X$ cross section at the Tevatron in Eq. (10). This value of $k_{\max }$ is also compatible with the scale $m_{\pi}$.

The conclusions of Ref. [19] were also challenged in Ref. [26]. They used an effective field theory with ultraviolet cutoff $\Lambda$ in which $X$ is treated as a charm-meson molecule. They calculated the inclusive prompt cross sections for producing $X$ in $p \bar{p}$ collisions at the Tevatron 
and in $p p$ collisions at the LHC using the event generators HERWIG and PYTHIA to calculate the production rate of $D^{*} \bar{D}$ at short distances and using the effective field theory to calculate the formation rate of the $X$ at long distances. With $\Lambda=100 \mathrm{MeV}$, their cross sections using PYTHIA were $0.05 \mathrm{nb}$ at the Tevatron and $0.04 \mathrm{nb}$ at the LHC, which are much smaller than the results from CDF and CMS in Eqs. (7) and (8). Their cross sections were compatible with the results from $\mathrm{CDF}$ and $\mathrm{CMS}$ for $\Lambda$ in the range from $500 \mathrm{MeV}$ to $1000 \mathrm{MeV}$. For these large ultraviolet cutoffs, the contributions from the charged-charm-meson-pair channels $D^{*+} D^{-}$and $D^{+} D^{*-}$ were larger than those from the $D^{* 0} \bar{D}^{0}$ and $D^{0} \bar{D}^{* 0}$ channels by about a factor of 2 .

The analysis in Ref. [26] was rejected in Ref. [29], whose authors included most of those of Ref. [19]. They argued that $k_{\max }$ must be determined "independently of any educated guesses on the explicit form" of $\psi_{X}(k)$. They did not address the issue that their derivation of the upper bound with $k_{\max }$ approximately equal to $\gamma_{X}$ fails for the explicit wave function in Eq. (2).

\section{ESTIMATE OF CROSS SECTION FOR $X$}

In this section, we derive an equality between the $X(3872)$ cross section and a charm-meson-pair cross section that takes into account the threshold enhancement produced by the $X$ resonance. We also present an order-ofmagnitude estimate of the $X$ cross section in terms of a naive charm-meson-pair cross section that does not take into account the threshold enhancement.

Expressions for the cross sections for producing charmmeson pairs and for producing $X$ in Eqs. (12) that take into account the $X$ resonance were presented in Ref. [20]. The cross sections were expressed in factored forms, with longdistance factors that involve the binding momentum $\gamma_{X}$ and with short-distance factors that involve only momentum scales of order $m_{\pi}$ or larger. The amplitude for producing $D^{* 0} \bar{D}^{0}+y$ in Eq. (12a) can be decomposed into chargeconjugation even $(C=+)$ and charge-conjugation odd $(C=-)$ components. The $C=+$ component is enhanced by the $X$ resonance. If the nonresonant $C=-$ component is neglected, the amplitude for producing $D^{* 0} \bar{D}^{0}+y$ can be expressed as a product of the $C=+$ component of a shortdistance amplitude and a resonance factor that depends on $\gamma_{X}$ :

$\mathcal{A}_{D^{* 0} \bar{D}^{0}+y}(\boldsymbol{k})=\frac{1}{\sqrt{2}}\left(\frac{\mathcal{A}_{D^{* 0} \bar{D}^{0}+y}^{\text {s.d. }}+\mathcal{A}_{D^{0} \bar{D}^{* 0}+y}^{\text {s.d. }}}{\sqrt{2}}\right) \frac{\Lambda}{-\gamma_{X}-i k}$.

The expression for the corresponding amplitude $\mathcal{A}_{D^{0} \bar{D}^{\star 0}+y}(\boldsymbol{k})$ is identical. The short-distance amplitudes $\mathcal{A}_{D^{* 0} \bar{D}^{0}+y}^{\text {s.d. }}$ and $\mathcal{A}_{D^{0} \bar{D}^{* 0}+y}^{\text {s.d. }}$ are independent of the momentum if $\boldsymbol{k}$ is small compared to $m_{\pi}$. The constant $\Lambda$ in the numerator of the resonance factor should be of order $m_{\pi}$. The only dependence on the small momentum $\gamma_{X}$ is in the denominator of the resonance factor. Since $\Lambda \gg \gamma_{X}$, the absolute value of the resonance factor is approximately 1 at $k=\Lambda$, so $\Lambda$ can be interpreted as the momentum scale where the amplitude becomes comparable in magnitude to the amplitude in the absence of the resonance. The resonance factor in Eq. (17) produces a threshold enhancement in the cross section. The differential cross section $d \sigma / d E$ in the kinetic energy $E$ of $D^{* 0} \bar{D}^{0}$ in the $D^{* 0} \bar{D}^{0}$ center-of-momentum frame should have a local minimum above the threshold enhancement. A simple physical interpretation of $\Lambda$ is that the kinetic energy $E$ at the local minimum is roughly $\Lambda^{2} / 2 \mu$.

The factorization formula for the $D^{* 0} \bar{D}^{0}$ cross section can be obtained simply by inserting the amplitude in Eq. (17) into Eq. (12a). The factorization formula for the $X$ cross section cannot be obtained so simply. If the universal wave function in Eq. (2) is inserted into Eq. (12c), the momentum integral is logarithmically ultraviolet divergent. The factorization formula for the $X$ cross section can be obtained instead by requiring the sum of the cross sections for producing $X$ and the cross sections for producing $D^{* 0} \bar{D}^{0}$ and $D^{0} \bar{D}^{* 0}$ integrated over $\boldsymbol{k}$ to be consistent with the optical theorem in Eq. (4). The resulting factorization formulas for the inclusive cross sections are

$$
\begin{aligned}
d \sigma\left[D^{* 0} \bar{D}^{0}\right]= & \frac{1}{\text { flux }} \sum_{y} \int d \Phi_{\left(D^{*} \bar{D}\right)+y} \mid \mathcal{A}_{D^{* 0} \bar{D}^{0}+y}^{\text {s.d. }} \\
& +\left.\mathcal{A}_{D^{D^{\circ}} \bar{D}^{* 0}+y}^{\text {sd }}\right|^{2} \frac{\Lambda^{2}}{\gamma_{X}^{2}+k^{2}} \frac{d^{3} k}{(2 \pi)^{3} 8 \mu}, \\
d \sigma\left[D^{0} \bar{D}^{* 0}\right]= & \frac{1}{\text { flux }} \sum_{y} \int d \Phi_{\left(D^{*} \bar{D}\right)+y} \mid \mathcal{A}_{D^{\text {so }} \bar{D}^{0}+y}^{\text {s.d. }} \\
& +\left.\mathcal{A}_{D^{0} \bar{D}^{* 0}+y}^{\text {s.d }}\right|^{2} \frac{\Lambda^{2}}{\gamma_{X}^{2}+k^{2}} \frac{d^{3} k}{(2 \pi)^{3} 8 \mu}, \\
d \sigma[X(3872)]= & \frac{1}{\text { flux }} \sum_{y} \int d \Phi_{\left(D^{*} \bar{D}\right)+y} \mid \mathcal{A}_{D^{* 0} \bar{D}^{0}+y}^{\text {s.d. }} \\
& +\left.\mathcal{A}_{D^{0} \bar{D}^{* 0}+y}^{\text {s.d. }}\right|^{2} \frac{\Lambda^{2} \gamma_{X}}{8 \pi \mu} .
\end{aligned}
$$

The differential cross sections for $D^{* 0} \bar{D}^{0}$ and $D^{0} \bar{D}^{* 0}$ in Eqs. (18a) and (18b) should be good approximations up to relative momentum $k$ of about $\Lambda$.

The short distance factors in Eqs. (18) can be eliminated to obtain an expression for the $D^{* 0} \bar{D}^{0}$ cross section in terms of the $X$ cross section:

$$
d \sigma\left[D^{* 0} \bar{D}^{0}\right]=d \sigma[X(3872)] \frac{\pi / \gamma_{X}}{\gamma_{X}^{2}+k^{2}} \frac{d^{3} k}{(2 \pi)^{3}} .
$$

This relation is analogous to relations between cross sections for the production of the deuteron bound state with large momentum transfer and cross sections for the 
production of proton-neutron pairs [30]. The integral of this $D^{* 0} \bar{D}^{0}$ cross section over the region $|\boldsymbol{k}|<k_{\max }$ is

$$
\begin{aligned}
& \sigma\left[D^{* 0} \bar{D}^{0}\left(k<k_{\max }\right)\right] \\
& \quad=\sigma[X(3872)] \frac{k_{\max } / \gamma_{X}-\arctan \left(k_{\max } / \gamma_{X}\right)}{2 \pi} .
\end{aligned}
$$

There is a value of $k_{\max }$ such that the integrated cross section is equal to that for $X: k_{\max }=7.73 \gamma_{X}$. The resulting equality between the $X$ cross section and a $D^{* 0} \bar{D}^{0}$ cross section is

$$
\sigma[X(3872)]=\sigma\left[D^{* 0} \bar{D}^{0}\left(k<7.73 \gamma_{X}\right)\right] .
$$

The right side of this equality can also be expressed as the sum of the $D^{* 0} \bar{D}^{0}$ and $D^{0} \bar{D}^{* 0}$ cross sections integrated up to a smaller maximum value of $k$ :

$$
\sigma[X(3872)]=2 \sigma\left[D^{* 0} \bar{D}^{0}\left(k<4.49 \gamma_{X}\right)\right] .
$$

The equalities in Eqs. (21) and (22) are equivalent provided $\gamma_{X}$ is sufficiently small. As a reasonable condition for the validity of the equality, we require $k_{\max }$ to be smaller than the inverse range $m_{\pi}$. If this condition is applied to the equalities in Eqs. (21) and (22), it requires the binding energy $\left|E_{X}\right|$ to be less than about 0.2 and $0.5 \mathrm{MeV}$, respectively.

The equality in Eq. (21) is consistent with the upper bound in Eq. (15) for any value of $k_{\max }$ greater than $7.73 \gamma_{X}$. However the derivation of the upper bound in Eq. (15) requires $k_{\max }$ to be order $m_{\pi}$, because the derivation must allow for the possibility that the wave function for $X$ has the form in Eq. (2). The equality in Eq. (21) is incompatible with the upper bound in Eq. (15) if $k_{\max }$ is taken to be approximately $\gamma_{X}$, as was apparently assumed in Ref. [19].

The equality between the cross sections for $X$ and $D^{* 0} \bar{D}^{0}$ in Eq. (21) assumes the $D^{* 0} \bar{D}^{0}$ cross section has the threshold enhancement from the $X$ resonance. If an event generator such as HERWIG or PYTHIA is used to estimate the charm-meson-pair cross section, the equality in Eq. (21) cannot be used because the event generator is not informed about the resonance. The naive cross section for producing $D^{* 0} \bar{D}^{0}$ can be obtained from Eq. (12a) by replacing the amplitude $\mathcal{A}_{D^{* 0} \bar{D}^{0}+y}(\boldsymbol{k})$ by the short-distance amplitude $\mathcal{A}_{D^{* 0} \bar{D}^{0}+y}^{\text {s.d. }}$

$d \sigma\left[D^{* 0} \bar{D}^{0}\right]_{\text {naive }} \approx \frac{1}{\text { flux }} \sum_{y} \int d \Phi_{\left(D^{*} \bar{D}\right)+y}\left|\mathcal{A}_{D^{* 0} \bar{D}^{0}+y}^{\text {s.d. }}\right|^{2} \frac{d^{3} k}{(2 \pi)^{3} 2 \mu}$.

This naive $D^{* 0} \bar{D}^{0}$ cross section integrated over the region $|\boldsymbol{k}|<k_{\max }$ scales like $k_{\max }^{3}$, in agreement with the charmmeson cross section calculated using an event generator in
Ref. [20]. In the expression for the $X$ cross section in Eq. (18c), the sum over the many additional particles $y$ give cancellations that suppress the interference terms between the amplitudes $\mathcal{A}_{D^{* 0} \bar{D}^{0}+y}^{\text {s.d. }}$ and $\mathcal{A}_{D^{0} \bar{D}^{* 0}+y}^{\text {s.d. }}$. In a high energy hadron collider, the production rates for $D^{* 0} \bar{D}^{0}$ and $D^{0} \bar{D}^{* 0}$ at large transverse momentum should be equal, because the light quarks in the charm mesons are unlikely to come from the colliding hadrons. The $D^{* 0} \bar{D}^{0}$ and $D^{0} \bar{D}^{* 0}$ contributions should therefore be equal, so the cross section reduces to

$d \sigma[X(3872)]=\frac{1}{\text { flux }} \sum_{y} \int d \Phi_{\left(D^{*} \bar{D}\right)+y}\left|\mathcal{A}_{D^{* 0} \bar{D}^{0}+y}^{\text {s.t. }}\right|^{2} \frac{\Lambda^{2} \gamma_{X}}{4 \pi \mu}$.

The short distance factor in the expression for the naive $D^{* 0} \bar{D}^{0}$ cross section in Eq. (23) can then be eliminated in favor of the $X$ cross section using Eq. (24):

$$
d \sigma\left[D^{* 0} \bar{D}^{0}\right]_{\text {naive }} \approx d \sigma[X(3872)] \frac{2 \pi / \gamma_{X}}{\Lambda^{2}} \frac{d^{3} k}{(2 \pi)^{3}} .
$$

Note that this is larger by a factor of 2 than the cross section obtained from the equality in Eq. (19) by replacing $\gamma_{X}^{2}+k^{2}$ in the denominator by $\Lambda^{2}$. If the naive $D^{* 0} \bar{D}^{0}$ cross section in Eq. (25) is integrated over the region $|\boldsymbol{k}|<k_{\max }$, there is a value of $k_{\max }$ for which the integrated cross section is equal to the $X$ cross section:

$$
\sigma[X(3872)] \approx \sigma_{\text {naive }}\left[D^{* 0} \bar{D}^{0}\left(k<\left(3 \pi \Lambda^{2} \gamma_{X}\right)^{1 / 3}\right)\right] .
$$

This result can be used to obtain an order-of-magnitude estimate of the $X$ cross section using naive charm-mesonpair cross sections calculated using a Monte Carlo event generator. Since $\Lambda$ is order $m_{\pi}$, this estimate is compatible with the upper bound in Eq. (15) with $k_{\max }$ of order $m_{\pi}$.

In Ref. [20], the estimate of the $X$ cross section in terms of a naive charm-meson-pair cross section was expressed in the form

$$
\sigma[X(3872)] \approx \sigma_{\text {naive }}\left[D^{* 0} \bar{D}^{0}(k<\Lambda)\right] \frac{6 \pi \gamma_{X}}{\Lambda} .
$$

The naive $D^{* 0} \bar{D}^{0}$ cross section integrated up to a relative momentum $k_{\max }$ that was calculated using the PYTHIA event generator in Ref. [20] scales as $k_{\max }^{3}$. The estimate in Eq. (27) therefore differs from that in Eq. (26) only by a multiplicative factor of 2. Thus the estimate in Eq. (26) is essentially just a convenient repackaging of the estimate from Ref. [20] in Eq. (27).

The estimate of the $X$ cross section in Eq. (26) depends on the combination $\left(\Lambda^{2} \gamma_{X}\right)^{1 / 3}$ of unknown parameters. If we use the lower bound on the $X$ cross section at the Tevatron in Eq. (10) and the naive estimate of the $D^{* 0} \bar{D}^{0}$ cross section at the Tevatron in Eq. (16), we get the lower 
bound $\left(\Lambda^{2} \gamma_{X}\right)^{1 / 3}>100 \mathrm{MeV}$. The order-of-magnitude estimate of this combination of parameters obtained by inserting $\Lambda=m_{\pi}$ and $\gamma_{X}=18 \mathrm{MeV}$ is $\left(\Lambda^{2} \gamma_{X}\right)^{1 / 3} \sim$ $70 \mathrm{MeV}$. We conclude that the estimate of the $X$ cross section at the Tevatron in Eq. (27) is compatible with the observed cross section for some value of $\Lambda$ of order $m_{\pi}$ if the binding energy of the $X$ is roughly $0.17 \mathrm{MeV}$.

There does not seem to be any calculation using event generators in the literature of the naive cross section for producing $D^{* 0} \bar{D}^{0}$ with small relative momentum at the LHC. If there was such a calculation for a single small value of $k_{\max }$, the cross section as a function of $k_{\max }$ could be obtained simply by assuming it scales as $k_{\max }^{3}$. Equation (26) would then give an estimate of the cross section for producing $X$ at the LHC that could be compared with the measured value in Eq. (11). Given the large uncertainty in $k_{\max }=\left(3 \pi \Lambda^{2} \gamma_{X}\right)^{1 / 3}$ and the fact that the estimate of the cross section scales as $k_{\max }^{3}$, there is little doubt that the estimate would be compatible with the observed cross section.

\section{SUMMARY AND DISCUSSION}

We have discussed the inclusive prompt production of the $X(3872)$ at high energy hadron colliders under the assumption that the $X$ is a weakly bound charm-meson molecule with the particle content in Eq. (1). We considered the production of $X$ through the creation of its constituents $D^{* 0} \bar{D}^{0}$ or $D^{0} \bar{D}^{* 0}$ at short distances of order $1 / m_{\pi}$ or smaller. The formation of the $X$ proceeds on longer distance scales of order $1 / \gamma_{X}$, where $\gamma_{X}$ is the binding momentum of the $X$.

The theoretical upper bound on the cross section for producing $X$ in Eq. (15) was derived in Ref. [19]. It is given by the $D^{* 0} \bar{D}^{0}$ cross section integrated up to relative momentum $k_{\max }$. The authors did not give any clear prescription for $k_{\max }$, but their numerical value for $k_{\max }$ was consistent with it being approximately equal to $\gamma_{X}$. In Ref. [20], it was pointed out that the derivation of the upper bound in Eq. (15) actually requires $k_{\max }$ to be of order $m_{\pi}$. A specific example of a wave function for which the derivation of the upper bound requires $k_{\max } \gg \gamma_{X}$ is the universal wave function for a weakly bound molecule in Eq. (2). This failure of the derivation of their upper bound with $k_{\max }$ approximately equal to $\gamma_{X}$ has never been addressed by the authors of Ref. [19].

Assuming the binding energy of the $X$ is sufficiently small, we used the methods of Ref. [20] to derive the equality in Eq. (21) between the $X$ cross section and the $D^{* 0} \bar{D}^{0}$ cross section integrated up to relative momentum $k_{\max }=7.73 \gamma_{X}$. This equality takes into account the threshold enhancement in the charm-meson-pair cross section associated with the $X$ resonance. The condition for the validity of this equality is that the binding energy $\left|E_{X}\right|$ is less than about $0.2 \mathrm{MeV}$.

The equality in Eq. (21) is not applicable if the charmmeson-pair cross section is estimated using a naive method that is not informed about the resonance, such as a Monte Carlo event generator. We used the methods of Ref. [20] to derive the order-of-magnitude estimate for the $X$ cross section in Eq. (26). It is expressed as the naive $D^{* 0} \bar{D}^{0}$ cross section integrated up to a relative momentum $k_{\max }$ of order $\left(m_{\pi}^{2} \gamma_{X}\right)^{1 / 3}$. The resulting estimate for the prompt cross section for $X$ at the Tevatron using the naive $D^{* 0} \bar{D}^{0}$ cross section in Eq. (16) is compatible with the experimental lower bound on the prompt $X$ cross section given in Eq. (10). Given the large uncertainty in $k_{\max }$ and the $k_{\max }^{3}$ scaling of the naive cross section, there is little doubt that the corresponding estimate of the cross section at the LHC would be compatible with the measured value in Eq. (11). We conclude that the prompt cross sections for $X$ at the Tevatron and at the LHC are compatible with the identification of $X(3872)$ as a weakly bound charm-meson molecule.

\section{ACKNOWLEDGMENTS}

This work was supported in part by the Department of Energy under Grant No. DE-SC0011726 and by the National Science Foundation under Grant No. PHY1607190. We thank T. Skwarnicki and F. K. Guo for valuable comments.
[1] H. X. Chen, W. Chen, X. Liu, and S. L. Zhu, The hidden-charm pentaquark and tetraquark states, Phys. Rep. 639, 1 (2016).

[2] A. Hosaka, T. Iijima, K. Miyabayashi, Y. Sakai, and S. Yasui, Exotic hadrons with heavy flavors: $X, Y, Z$, and related states, Prog. Theor. Exp. Phys. 2016, 062 C01 (2016).

[3] R. F. Lebed, R. E. Mitchell, and E. S. Swanson, Heavyquark QCD exotica, Prog. Part. Nucl. Phys. 93, 143 (2017).

[4] A. Esposito, A. Pilloni, and A. D. Polosa, Multiquark resonances, Phys. Rep. 668, 1 (2017).
[5] F. K. Guo, C. Hanhart, U. G. Meißner, Q. Wang, Q. Zhao, and B.S. Zou, Hadronic molecules, Rev. Mod. Phys. 90, 015004 (2018).

[6] A. Ali, J. S. Lange, and S. Stone, Exotics: Heavy pentaquarks and tetraquarks, Prog. Part. Nucl. Phys. 97, 123 (2017).

[7] S. L. Olsen, T. Skwarnicki, and D. Zieminska, Nonstandard heavy mesons and baryons: Experimental evidence, Rev. Mod. Phys. 90, 015003 (2018). 
[8] M. Karliner, J. L. Rosner, and T. Skwarnicki, Multiquark states, Annu. Rev. Nucl. Part. Sci. 68, 17 (2018).

[9] C. Z. Yuan, The $X Y Z$ states revisited, Int. J. Mod. Phys. A 33, 1830018 (2018).

[10] N. Brambilla, S. Eidelman, C. Hanhart, A. Nefediev, C. P. Shen, C. E. Thomas, A. Vairo, and C.Z. Yuan, The XYZ states: Experimental and theoretical status and perspectives, arXiv:1907.07583.

[11] S. K. Choi et al. (Belle Collaboration), Observation of a Narrow Charmonium-Like State in Exclusive $B^{ \pm} \rightarrow$ $K^{ \pm} \pi^{+} \pi^{-} J / \psi$ Decays, Phys. Rev. Lett. 91, 262001 (2003).

[12] D. Acosta et al. (CDF Collaboration), Observation of the Narrow State $X(3872) \rightarrow J / \psi \pi^{+} \pi^{-}$in $\bar{p} p$ Collisions at $\sqrt{s}=1.96 \mathrm{TeV}$, Phys. Rev. Lett. 93, 072001 (2004).

[13] R. Aaij et al. (LHCb Collaboration), Determination of the $X(3872)$ Meson Quantum Numbers, Phys. Rev. Lett. 110, 222001 (2013).

[14] M. Tanabashi et al. (Particle Data Group), Review of particle physics, Phys. Rev. D 98, 030001 (2018).

[15] V. M. Abazov et al. (D0 Collaboration), Observation and Properties of the $X(3872)$ Decaying to $J / \psi \pi^{+} \pi^{-}$in $p \bar{p}$ Collisions at $\sqrt{s}=1.96 \mathrm{TeV}$, Phys. Rev. Lett. 93, 162002 (2004).

[16] R. Aaij et al. (LHCb Collaboration), Observation of $X(3872)$ production in $p p$ collisions at $\sqrt{s}=7 \mathrm{TeV}$, Eur. Phys. J. C 72, 1972 (2012).

[17] S. Chatrchyan et al. (CMS Collaboration), Measurement of the $X(3872)$ production cross section via decays to $J / \psi \pi \pi$ in $p p$ collisions at $\sqrt{s}=7 \mathrm{TeV}$, J. High Energy Phys. 04 (2013) 154.

[18] M. Aaboud et al. (ATLAS Collaboration), Measurements of $\psi(2 S)$ and $X(3872) \rightarrow J / \psi \pi^{+} \pi^{-}$production in $p p$ collisions at $\sqrt{s}=8 \mathrm{TeV}$ with the ATLAS detector, J. High Energy Phys. 01 (2017) 117.

[19] C. Bignamini, B. Grinstein, F. Piccinini, A. D. Polosa, and C. Sabelli, Is the $X(3872)$ Production Cross Section at
Tevatron Compatible with a Hadron Molecule Interpretation?, Phys. Rev. Lett. 103, 162001 (2009).

[20] P. Artoisenet and E. Braaten, Production of the $X(3872)$ at the Tevatron and the LHC, Phys. Rev. D 81, 114018 (2010).

[21] E. Braaten and H.-W. Hammer, Universality in few-body systems with large scattering length, Phys. Rep. 428, 259 (2006).

[22] G. Gokhroo et al. (Belle Collaboration), Observation of a Near-Threshold $D^{0} \bar{D}^{0} \pi^{0}$ Enhancement in $B \rightarrow D^{0} \bar{D}^{0} \pi^{0} K$ Decay, Phys. Rev. Lett. 97, 162002 (2006).

[23] G. Bauer, The $X(3872)$ meson and "exotic" spectroscopy at CDF II, Int. J. Mod. Phys. A 21, 959 (2006).

[24] E. Braaten, L.-P. He, and K. Ingles, Branching fractions of the $X(3872)$, arXiv:1908.02807.

[25] G. Wormser (BABAR Collaboration), Quarkonium 2019 in Torino (2019), https://agenda.infn.it/event/15632/ contributions/89320/attachments/63025/75778/charmonium_ QWG19.pdf.

[26] M. Albaladejo, F. K. Guo, C. Hanhart, U. G. Meißner, J. Nieves, A. Nogga, and Z. Yang, Note on X(3872) production at hadron colliders and its molecular structure, Chin. Phys. C 41, 121001 (2017).

[27] F. K. Guo, U. G. Meißner, W. Wang, and Z. Yang, Production of the bottom analogs and the spin partner of the $X(3872)$ at hadron colliders, Eur. Phys. J. C 74, 3063 (2014).

[28] S.-K. Choi et al., Bounds on the width, mass difference and other properties of $X(3872) \rightarrow \pi^{+} \pi^{-} J / \psi$ decays, Phys. Rev. D 84, 052004 (2011).

[29] A. Esposito, B. Grinstein, L. Maiani, F. Piccinini, A. Pilloni, A. D. Polosa, and V. Riquer, Comment on "Note on $X(3872)$ production at hadron colliders and its molecular structure, Chin. Phys. C 42, 114107 (2018).

[30] G. Faeldt and C. Wilkin, Bound state and continuum production in large momentum transfer reactions, Phys. Lett. B 382, 209 (1996). 